Revista Colombiana de Obstetricia y Ginecología Vol. 66 No. 1 • Enero-Marzo 2015 • (61-66)

\title{
EMBARAZO ABDOMINAL AVANZADO CON FETO VIVO. REPORTE DE UN CASO Y REVISIÓN DE LA LITERATURA
}

\section{Advanced abdominal pregnancy with a live foetus. Case report and review of the literature}

Samuel Gélvez-Téllez, $M D^{1}$

Recibido: julio 28/14 - Aceptado: febrero 23/15

\section{RESUMEN}

Objetivos: describir el caso de una paciente con embarazo abdominal a término y feto vivo, y realizar una revisión de la literatura sobre el diagnóstico y tratamiento, con énfasis en la extracción de la placenta y el uso de metotrexate para el manejo de la placenta residual.

Materiales y métodos: se presenta el caso de una paciente con un embarazo abdominal avanzado de 38 semanas y 5 días de gestación, atendida en un hospital público de referencia de mediana complejidad, ubicado en el departamento del Chocó, en la costa pacífica colombiana, quien fue llevada a cirugía por sufrimiento fetal agudo, feto en podálica y miomatosis uterina. Durante el procedimiento y como hallazgo incidental se encontró un embarazo abdominal, se obtuvo un feto vivo en buenas condiciones y se realizó extracción manual de la placenta, con buena evolución de la madre y el recién nacido. No se utilizó metotrexate como parte del manejo del tejido placentario residual. Se realizó una búsqueda bibliográfica en las bases de datos de Medli-

1. Ginecólogo y obstetra. Hospital San Francisco de Asís, Quibdó, Chocó (Colombia); Clínica Materno-infantil, Fundación SOMA, Chigorodó, Antioquia (Colombia).s_gelvez@yahoo.com ne vía PubMed, Embase y la Biblioteca Cochrane, utilizando las siguientes palabras clave: embarazo abdominal, feto vivo, metotrexate, placenta.

Resultados: en total se encontraron 31 artículos relacionados con el tema, de los cuales se seleccionaron 23 para la revisión: 18 eran reportes de casos, 4 artículos de revisión de la literatura y una carta al editor.

Conclusión: el embarazo abdominal avanzado es una rara entidad con alta tasa de morbi-mortalidad materna y perinatal. A pesar de los adelantos en la ultrasonografía, continúa siendo subdiagnosticado. Su manejo debe ser en un tercer nivel, con un equipo multidisciplinario y con disponibilidad de productos sanguíneos. Faltan estudios que permitan incluir y valoren la seguridad del metotrexate y de la extracción de la placenta en los protocolos de manejo en el embarazo abdominal avanzado.

Palabras clave: embarazo abdominal, feto vivo, metotrexate, placenta.

\section{ABSTRACT}

Objectives: To describe the case of a patient delivered at term of a live foetus after abdominal pregnancy, and to conduct a review of the literature on the diagnosis and treatment with emphasis on 
placental extraction and the use of methotrexate for the management of the residual placenta.

Materials and methods: Case presentation of a patient with advanced abdominal pregnancy of 38 weeks and 5 days of gestation seen at an intermediate complexity public referral hospital located in the Department of Chocó on the Pacific Coast of Colombia. The patient was taken to surgery due to acute foetal distress, breech presentation and uterine myomatosis. The abdominal pregnancy was found incidentally during the procedure. A live foetus was delivered in good condition, the placenta was removed manually and the mother and neonate evolved satisfactorily. Methotrexate was not used as part of the management of the residual placental tissue. A search of the literature was conducted in the Medline database through PubMed, Embase and The Cochrane Library, using the following key words: abdominal pregnancy, live foetus, methotrexate, placenta.

Results: At total of 31 articles on the topic were found and 23 were selected for the review: 18 case reports, 4 literature review articles and one letter to the editor.

Conclusion: Advanced abdominal pregnancy is a rare entity associated with high maternal and perinatal mortality and morbidity rates. Despite advances in ultrasound, this entity is still underdiagnosed. It must be managed in a level III hospital by a multidisciplinary team, with blood products available. Studies leading to the inclusion and assessment of the safety of methotrexate and placental extraction in the management protocols for advanced abdominal pregnancy are needed.

Key words: Abdominal pregnancy, live foetus, methotrexate, placenta.

\section{INTRODUCCIÓN}

El embarazo abdominal es un tipo especial de embarazo ectópico, cuya implantación se da a nivel extrauterino, dentro de la cavidad peritoneal y por fuera del ovario, la trompa o los ligamentos. Se clasifica en dos tipos: el primario, donde la implanta- ción del huevo fertilizado ocurre directamente en la cavidad abdominal, encontrando la trompa y los ovarios intactos, y el secundario, que comprende la mayoría de los casos, donde la implantación peritoneal ocurre posterior a la ruptura de la trompa de Falopio o por un aborto tubárico (1). Si bien la mayoría carece de una suficiente invasión trofoblástica para continuar manteniendo el embarazo, unos pocos podrían sobrevivir y progresar más allá del segundo trimestre (2). Cuando alcanza las 18 o más semanas de gestación, se denomina embarazo abdominal avanzado (2). La prevalencia del embarazo abdominal avanzado se ha calculado en 1 de cada 25.000 embarazos (3), con una tasa de mortalidad materna entre el 0,5 y el 8,5\%, cerca de noventa veces la mortalidad materna asociada a un embarazo normal (4). Se ha reportado una mortalidad perinatal entre el 40 y el $95 \%(5,6)$.

No obstante su baja prevalencia, cada vez hay más publicaciones que informan de un embarazo abdominal avanzado (7-9); se ha encontrado que esta complicación del embarazo ha aumentado su frecuencia con los procedimientos de fertilización in vitro (10), los antecedentes de cirugía uterina y la dilatación y curetaje $(11,12)$, incrementando la probabilidad de que en algún momento de nuestra práctica nos encontremos con una paciente que presente esta condición.

Históricamente, el departamento del Chocó se ha caracterizado por presentar una alta proporción de personas con necesidades básicas insatisfechas (70\%) y una razón de mortalidad materna de 180 x 100.000 nacidos vivos (13), con un $13 \%$ de mujeres que, según la Encuesta Nacional de Demografía y Salud, Colombia 2010, no asistió a ningún control prenatal (14). Esta situación es frecuente en algunas regiones en Colombia y Latinoamérica donde hay limitaciones en la disponibilidad de tecnologías que hacen compleja la práctica de la obstetricia, en especial en la detección y el manejo de condiciones como la del caso que se presenta. Por otra parte, hay controversia en cuanto a la seguridad de la extracción de la placenta y uso del 
metotrexate en el manejo de la misma. Se presenta este caso de embarazo abdominal avanzado con el objetivo realizar una revisión de la literatura en el diagnóstico y tratamiento, haciendo énfasis en la extracción de la placenta y el uso de metotrexate para el manejo de la placenta residual.

\section{REPORTE DE CASO}

Paciente de 34 años de edad, multigestante, grávida: 4, partos: 3, vivos: 3, abortos: 0, cesáreas: 0 , residente en área rural, con ingreso tardío al control prenatal, con embarazo de 38 semanas y 5 días de edad gestacional por fecha de última menstruación, y ecografía del tercer trimestre, sin ecografías tempranas, que es remitida al hospital público San Francisco de Asís de Quibdó, única institución de segundo nivel del departamento del Chocó. Presentaba cuadro clínico de dos días de evolución de disminución de los movimientos fetales, no refería actividad uterina. En la ecografía realizada a las 34 semanas de gestación, reportaban feto único, vivo, en podálica, ILA de 4 y placenta corporal posterior grado 2. No describían presentación ectópica; además, referían una masa compatible con mioma gigante que ocupaba la parte derecha del abdomen materno. Al ingreso se encontró una paciente en buenas condiciones generales, a nivel abdominal no se palparon masas, altura uterina de $29 \mathrm{~cm}$, feto en podálica, sin actividad uterina, leve dolor a la palpación abdominal, frecuencia cardiaca fetal de 105 lpm; al tacto vaginal se encontró un cérvix posterior largo y cerrado. Se hospitalizó para estudio y manejo de su condición; se ordenó monitorización fetal, la cual mostró bradicardia, variabilidad disminuida y desaceleraciones espontáneas, por lo que se programó para cesárea urgente con diagnóstico de sufrimiento fetal agudo, feto en podálica y miomatosis uterina.

Se inició el procedimiento con una incisión tipo pfannenstiel, la cual se convirtió a mediana para una mejor exposición del campo quirúrgico, se identificó una gran masa encapsulada que ocupaba todo el abdomen, adherida al epiplón e intestino, y muy vascularizada; dentro de esta se hallaban el feto y la placenta; a nivel de la fosa pélvica se observó el útero con ubicación, consistencia y tamaño normales. Se incide la masa encapsulada y se extrae el feto, que presentaba asimetría facial del lado izquierdo, adaptación adecuada, con peso de $3.100 \mathrm{~g}$, el cual se entregó al equipo de pediatría que continuó el manejo neonatal; posteriormente, se procedió a la remoción de la placenta, disecando en los sitios de unión con las estructuras abdominales; se realizó adhesiólisis y disección de vasos neoformados, se extrajo la placenta casi en su totalidad, quedando algunas áreas de tejido placentario adheridas a las asas intestinales. Durante el procedimiento presentó sangrado en capa en los sitios de disección y de liberación de las adherencias, que se controló fácilmente con electrocoagulación; se revisó la cavidad abdominal y no se encontraron lesiones de estructuras vecinas. Debido al sangrado se transfundió intraquirúrgicamente con dos unidades de glóbulos rojos. El procedimiento se terminó sin complicaciones. No se utilizó tratamiento médico con metotrexate, la evolución posoperatoria fue adecuada y se dio de alta a la madre y al feto al quinto día posquirúrgico; se revisó nuevamente a la paciente cada semana por un mes, y luego a los dos y seis meses posoperatorios, encontrándose en buenas condiciones.

Aspectos éticos. La paciente autorizó la publicación del caso. Se garantizó total confidencialidad de la información y datos de la paciente.

\section{MATERIALES Y MÉTODOS}

Se realizó una búsqueda bibliográfica en las bases de datos de Medline vía PubMed, Embase y la Biblioteca Cochrane, en inglés y español, durante el periodo comprendido entre enero de 1970 hasta diciembre de 2014; se utilizaron los términos embarazo abdominal, feto vivo, placenta y metotrexate, se incluyeron artículos de revisión, reporte de casos y cartas al editor.

\section{RESULTADOS}

En total se encontraron 31 artículos relacionados con el tema, de los cuales se seleccionaron 23: 18 
eran reportes de casos, 4 artículos de revisión de la literatura y una carta el editor.

Diagnóstico. El diagnóstico de embarazo abdominal avanzado requiere un alto índice de sospecha, la mayoría de los síntomas son inespecíficos y muchas pacientes son asintomáticas. La paciente puede referir dolor abdominal tipo calambre, náuseas, vómito, malestar, movimientos fetales dolorosos, se puede encontrar aumento de la sensibilidad abdominal, partes fetales fácilmente palpables (15) y posición fetal anómala. La ausencia de contracciones durante la estimulación con oxitocina podría alertarnos sobre un posible embarazo abdominal (16). Se ha reportado aumento de los niveles de alfafetoproteína (AFP) en este tipo de embarazo (2).

El diagnóstico de embarazo abdominal avanzado frecuentemente es incidental. En una revisión de 163 casos, solo el $45 \%$ fueron diagnosticados antes del parto (17). En 4 de 10 mujeres de la serie del hospital Parkland el diagnóstico fue realizado al momento de una cesárea ordenada por inducción fallida (2).

La ecografía es la principal herramienta para su diagnóstico $(1,2,15)$, pero aun con la tecnología actual tan solo se identifica en el 40 al $60 \%$ de las veces $(2,17)$. Stanley et al. (16) publicaron una serie de hallazgos ecográficos encontrados en el embarazo abdominal: útero vacío separado del feto en el $90 \%$ de las pacientes, placenta ectópica en el $75 \%$, oligoamnios en el $45 \%$, partes fetales en estrecha proximidad a la pared materna en el $45 \%$, presentación anormal del feto en el $25 \%$, pobre visualización de la placenta en el $25 \%$, falla para visualizar el miometrio entre el feto y la vejiga materna en el $15 \%$, porcentajes que contrastan con los datos más recientes, pues la identificación de un útero vacío con un feto adyacente a este es reportada en menos del $30 \%$ de los casos $(2,17)$, los cuales se encontraron principalmente en ecografías realizadas antes de la semana 20 de gestación (2).

En la paciente hemodinámicamente estable, además de ayudar al diagnóstico, la resonancia magnética proporciona información adicional sobre la implantación placentaria y su relación con los vasos, tejidos y órganos adyacente $(18,19)$, lo que favorece la planeación quirúrgica.

Tratamiento. Dependerá del momento del diagnóstico y del nivel de atención, el cual idealmente debe ser en instituciones de alta complejidad. Los objetivos principales incluyen la extracción del feto y una adecuada evaluación y manejo de la implantación vascular y placentaria (2). La extracción de un feto vivo a término es rara, con pocos casos publicados en la literatura $(7,20,21)$.

Independientemente de la localización de la placenta, la disección quirúrgica es difícil y la hemorragia es común, con necesidad de transfusión de glóbulos rojos hasta en el $90 \%$ de las pacientes (2). Una vez la implantación placentaria ha sido evaluada, se debe considerar la realización de procedimientos que ayuden a disminuir el daño intraoperatorio, como la embolización angiográfica (15), la inserción de un catéter ureteral y una adecuada preparación intestinal (22). La decisión de remoción de la masa placentaria dependerá de la vascularización, de la extensión de la placentación, del compromiso intestinal y de la experiencia del equipo quirúrgico (23). La mayoría de los autores coinciden en que la placenta debería ser removida solo si es posible identificar y ligar el soporte sanguíneo sin daño a otros órganos; en caso de que esto no sea posible, es mejor no extraerla y posteriormente hacer vigilancia estricta para detectar posibles complicaciones (17).

Nohay consenso en la utilización de medicamentos para el tratamiento del tejido placentario. Se han utilizado diferentes esquemas con metotrexate para el manejo de la placenta o tejido placentario no removido con resultados variables (20). Huang et al. (23) reportaron una disminución adecuada de los niveles de gonadotropina, sin complicaciones asociadas al metotrexate durante el seguimiento de la paciente, con resultados similares a lo reportado por Mora-Enríquez et al. (7) y Valenzano et al. (24).

Aunque el metotrexate puede reducir directamente los niveles de BHCG, su uso inmediato después de la cirugía puede llevar a una rápida necrosis lobular placentaria, lo que facilita el 
crecimiento bacteriano y el aumento del riesgo de sepsis y muerte (23). Rahman et al. describieron cinco pacientes tratados en el posoperatorio con metotrexate, y si bien hubo una disminución rápida de los niveles de gonadotropina, las cinco desarrollaron infección intraabdominal y dos murieron (19). Oztequin reportó el caso de una paciente en la cual se usó metotrexate, pero esta murió a la tercera semana por sepsis e insuficiencia cardiopulmonar (25).

Debido a que el mecanismo de acción consiste en inhibir rápidamente la división celular, el metotrexate muy probablemente tenga pocos efectos sobre la placenta madura, la cual tiene una limitada actividad proliferativa (2). Con o sin su utilización, la placenta frecuentemente presentará sobreinfección y requerirá remoción quirúrgica en una segunda intervención $(19,23)$. Por estas razones, varios autores desaconsejan el uso del metotrexate como complemento del manejo de la placenta en el embarazo abdominal avanzado $(15,25)$.

Igualmente, hay reportes de casos en los cuales no se usó metotrexate para el manejo de la placenta. Nkusu (17) reportó el caso de un embarazo abdominal avanzado con evolución favorable de la paciente, en el cual hubo tejido placentario residual y no se usó metotrexate. Marcellin et al. (8), realizaron manejo expectante de la placenta, no hubo remoción, no usaron metotrexate y después de 15 meses de seguimiento esta era indetectable en los controles ecográficos.

Nuestro caso se suma a los reportes en los cuales no se usó metotrexate y hubo una evolución posquirúrgica favorable, con feto vivo y a término.

\section{CONCLUSIÓN}

A pesar de los avances en la calidad de los equipos de ultrasonografía, la mayoría de los diagnósticos de embarazo abdominal avanzado son incidentales en el momento de una cesárea ordenada por cualquier otra indicación obstétrica; la mejor oportunidad para el diagnóstico sigue siendo la ecografía temprana, donde es posible una mejor identificación de los ha- llazgos del embarazo abdominal avanzado. Una vez realizado el diagnóstico, se debe referir a la paciente a un nivel de atención que cuente con todos los recursos disponibles. Algunos grupos no aconsejan el uso del metotrexate debido a los efectos adversos potencialmente catastróficos, incluida muerte por sepsis; otros lo han utilizado con resultados satisfactorios. Faltan estudios que permitan incorporar o rechazar el uso del metotrexate o la extracción de la placenta como práctica rutinaria en los protocolos de manejo de la misma en estas pacientes.

\section{REFERENCIAS}

1. Dahiya K, Sharma D. Advanced abdominal pregnancy: a diagnostic and management dilema. J Gynecol Surg. 2007;23:69-72.

2. Worley KC, Hnat MD, Cunningham FG. Advanced extrauterine pregnancy: diagnostic and therapeutic challenges. Am J Obstet Gynecol. 2008;198:297.e1-7.

3. Desai BR, Patted Shobhana S, Pujar Yeshita V, Ruge J. Advanced secondary abdominal pregnancy following rupture of rudimentary horn. J Obstet Gynecol India. 2005;55:180.

4. Atrash HK, Friede A, Hogue CJ. Abdominal pregnancy in the United States: frequency and maternal mortality. Obstet Gynecol. 1987;69:333-7.

5. Stevens CA. Malformations and deformations in abdominal pregnancy. Am J Med Genet. 1993;47: 1189-95.

6. Cotlar AM. Extrauterine pregnancy: a historical review. Curr Surg. 2000;57:484-92.

7. Mora-Enríquez JA, Arreaza-Graterol M, NossaMoreno HH, Rodríguez-OrtizJA. Embarazo abdominal avanzado: diagnóstico y manejo. Reporte de un caso y revisión de la literatura. Rev Colomb Obstet Ginecol. 2014;65;256-61.

8. Marcellin L, Ménard S, Lamau MC, Mignon A, Aubelle MS, Grangé G, et al. Conservative management of an advanced abdominal pregnancy at 22 weeks. AJP Rep. 2014;4:55-60.

9. Dabiri T, Marroquin GA, Bendek B, Agamasu E, Mikhail M. Advanced Extrauterine Pregnancy at 33 Weeks with a Healthy Newborn. BioMed Research International. 2014:102479. 
10. Moonen-Delarue MW, Haest JW. Ectopic pregnancy three times in line of which two advanced abdominal pregnancies. Eur J Obstet Gynecol Reprod Biol. 1996;66:87-8.

11. Roberts RV, Dickinson JE, Leung Y, Charles AK. Advanced abdominal pregnancy: still an occurrence in modern medicine. Aust N Z J Obstet Gynaecol. 2005;45:518-21.

12. Hyvarinen M, Raudaskoski T, Tekay A, Herva R. [Abdominal pregnancy]. Duodecim. 2009;125: 244851. Finnish.

13. Ministerio de Salud y Protección Social Colombia. Análisis de situación de salud según regiones Colombia 2013. [Visitado 2015 Feb 20]. Disponible en: https://www.minsalud.gov.co/Documentos\%20 y\%20Publicaciones/An\%C3\%A1lisis\%20de\%20 situaci\%C3\%B3n\%20de\%20salud\%20por\%20 regiones.pdf.

14. Profamilia. Encuesta Nacional de Demografía y Salud, Colombia 2010. Salud Materna Infantil. [Visitado 2015 Feb 20]. Disponible en: http://www. profamilia.org.co/encuestas/Profamilia/Profamilia/ index.php?option $=$ com_content\&view $=$ article\&id $=62$ \&Itemid $=9$

15. Dahab AA, Aburass R, Shawkat W, Babgi R, Essa O, Mujallid RH. Full-term extrauterine abdominal pregnancy: a case report. J Med Case Rep. 2011;5:531.

16. Stanley JH, Horger EO 3rd, Fagan CJ, Andriole JG, Fleischer AC. Sonographic findings in abdominal pregnancy. AJR Am J Roentgenol. 1986;147:1043-6.

17. Nkusu Nunyalulendho D, Einterz EM. Advanced abdominal pregnancy: case report and review of 163 cases reported since 1946. Rural Remote Health. 2008;8:1087.

18. Cotter AM, Jacques EG, Izquierdo LA. Extended field of view sonography: a useful tool in the diagnosis and management of abdominal pregnancy. J Clin Ultrasound. 2004;32:207-10.

19. Rahman MS, Al-Suleiman SA, Rahman J, Al-Sibai MH. Advanced abdominal pregnancy-observations in 10 cases. Obstet Gynecol. 1982;59:366-72.

20. Varma R, Mascarenhas L, James D. Successful outcome of advanced abdominal pregnancy with exclusive omental insertion. Ultrasound Obstet Gynecol. 2003;21:192-4.

21. Meseci E, Guzel Y, Zemheri E, Eser SK, Ozkanli S, Kumru P. A 34-week ovarian pregnancy: case report and review of the literature. J Turk Ger Gynecol Assoc. 2013;14:246-9.

22. Rahaman J, Berkowitz R, Mitty H, Gaddipati S, Brown B, Nezhat F. Minimally invasive management of an advanced abdominal pregnancy. Obstet Gynecol. 2004;103:1064-8.

23. Huang K, Song L, Wang L, Gao Z, Meng Y, Lu Y. Advanced abdominal pregnancy: an increasingly challenging clinical concern for obstetricians. Int J Clin Exp Pathol 2014;7:5461-72.

24. Valenzano M, Nicoletti L, Odicino F, Cocuccio S, Lorenzi P, Ragni N. Five-year follow-up of placental involution after abdominal pregnancy. J Clin Ultrasound. 2003;31:39-43.

25. Oztequin O. Letters to Editor. Australian and New Zealand Journal of Obstetrics and Gynaecology. 2006;46:261-4. 